\title{
The Meaning of SARS-CoV-2 Antibodies in a Patient with a Systemic Reaction to the mRNA-1273 SARS-CoV-2 Vaccine after Previous Natural Immunization
}

\author{
Lorraine Sazgary, Andrea Meienberg, Christoph T Berger, Michael Mayr \\ Medical Outpatient Clinic, University Hospital Basel, University of Basel, Basel, Switzerland
}

Received: 06/05/2021

Accepted: 07/05/2021

Published: 04/06/2021

\begin{abstract}
How to cite this article: Sazgary L, Meienberg A, Berger CT, Mayr M. The meaning of SARS-CoV-2 antibodies in a patient with a systemic rection to the mRNA-1273 SARS-CoV-2 vaccine after previous natural immunization. EJCRIM 2021;8: doi:10.12890/2021_002617.
\end{abstract}

Conflicts of Interests: The authors declare there are no competing interests.

This article is licensed under a Commons Attribution Non-Commercial 4.0 License

\section{ABSTRACT}

Objectives: There is limited experience regarding the meaning of SARS-CoV-2 antibodies after vaccination in patients with naturally acquired immunity.

Methods: We describe the case of a patient who received the first dose of the mRNA-1273 SARS-CoV-2 vaccine 6 months after his recovery from moderately severe COVID-19.

Results: Our patient had a positive nucleocapsid SARS-CoV-2 IgG/lgM titre with 78.7 multiple of cut-off indicating persistent humoral immune response 6 months after infection. After vaccination, he developed prolonged systemic symptoms (fever, fatigue, nausea, diarrhoea and myalgia) for a duration of 6 days.

Conclusion: SARS-CoV-2 nucleocapsid antibodies provide information about naturally acquired immunity. For the assessment of immune response to vaccination, measurement of the SARS-CoV-2 spike antibody titre before and after vaccination is essential. Patients with naturally acquired immunity might develop a prolonged systemic reaction to the first dose of the mRNA-1273 SARS-CoV-2 vaccine.

\section{LEARNING POINTS}

- Patients with naturally acquired immunity might develop a prolonged systemic reaction after receiving an mRNA SARS-CoV-2 vaccine.

- Depending on the clinical situation of SARS-CoV-2 infection and/or vaccination, different antibody titres should be determined.

- The SARS-CoV-2 nucleocapsid antibodies provide information on whether or not natural immunization has taken place. To quantify the immune response induced by vaccination, the SARS-CoV-2 spike antibody titre before and after vaccination has to be measured.

\section{KEYWORDS}

COVID-19, vaccine, immune-reactivity, SARS-CoV-2 nucleocapsid antibody, SARS-CoV-2 spike antibody

\section{CASE DESCRIPTION}

In July 2020, a 79-year-old man presented to the emergency department with a 7-day history of productive cough, subfebrile temperatures, fatigue and progressive dyspnoea. On admission, the patient was afebrile, his blood pressure was $162 / 94 \mathrm{mmHg}$, heart rate was $66 \mathrm{bpm}$, and oxygen saturation was $96 \%$ on room air. On physical examination, he presented with crackles over the lower lung fields and left-sided abdominal rebound tenderness. A nasopharyngeal swab for SARS-CoV-2 confirmed active infection. Blood tests were solely remarkable for 
chronic anaemia (Table 1). CT scans of the chest showed ground glass opacities predominantly in the lower lobes, affecting $4 \%$ of the total lung volume (Fig. 1). The patient was discharged from the emergency department with symptomatic treatment.

\begin{tabular}{|c|c|c|}
\hline $\begin{array}{l}\text { Laboratory value and } \\
\text { normal range }\end{array}$ & At COVID-19 diagnosis & $\begin{array}{l}4 \text { Days after first dose of } \\
\text { mRNA-1273 SARS-CoV-2 } \\
\text { vaccine }\end{array}$ \\
\hline WBC $\left(3.5-10.0 \times 10^{\circ} / 1\right)$ & 5.73 & 10.44 \\
\hline $\mathrm{Hb}(140-180 \mathrm{~g} / \mathrm{l})$ & 120 & 122 \\
\hline 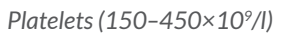 & 238 & 229 \\
\hline Neutrophils $\left(1.3-6.7 \times 10^{9} / 1\right)$ & 2.87 & 7.94 \\
\hline 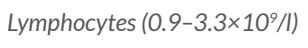 & 2.14 & 1.56 \\
\hline$C R P(<10 \mathrm{mg} / \mathrm{l})$ & 6.4 & 22.5 \\
\hline Creatine kinase (50-200 U/I) & 63 & 87 \\
\hline $\begin{array}{l}\text { Lactate dehydrogenase } \\
(135-225 \mathrm{U} / \mathrm{I})\end{array}$ & 191 & 212 \\
\hline Creatinine $(49-97 \mu \mathrm{mol} / \mathrm{l})$ & 79 & 84 \\
\hline Urea (3.4-8.7 mmol/l) & 6.3 & 6.5 \\
\hline
\end{tabular}

Table 1. Laboratory values at COVID-19 diagnosis and 4 days after the first dose of the mRNA-1273 SARS-CoV-2 vaccine

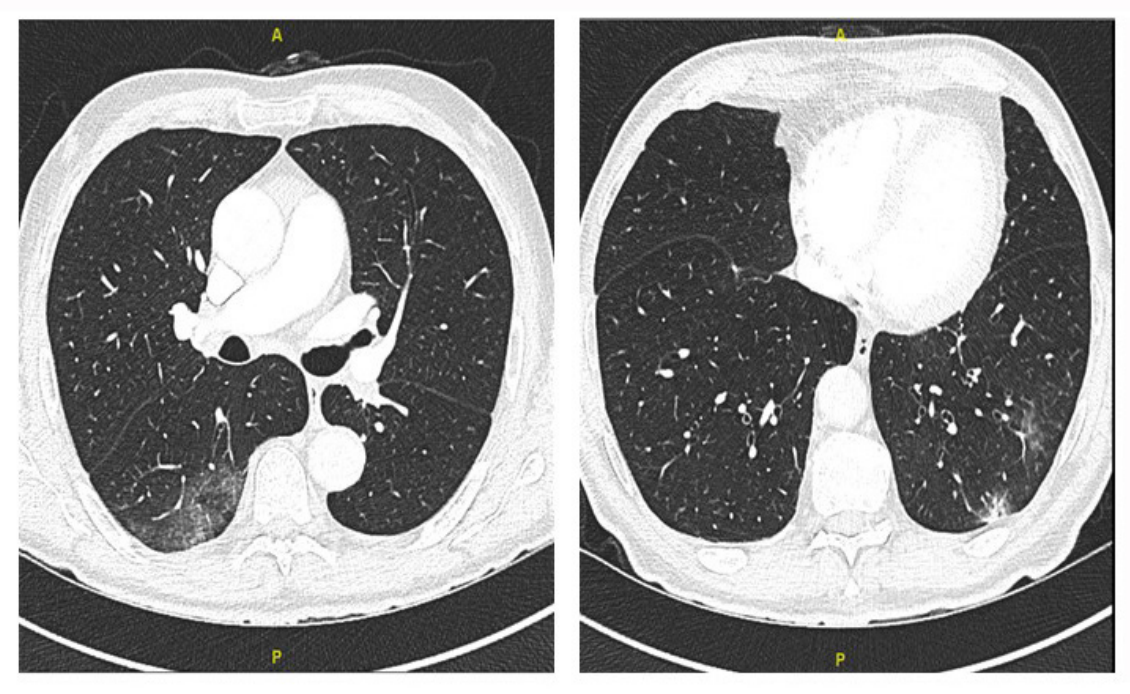

Figure 1. CT of the thorax: ground glass opacities are seen in the left and right lower lobes affecting $4 \%$ of the total lung volume

Six months after recovery from COVID-19, the patient received the first dose of the mRNA-1273 SARS-CoV-2 vaccine (Moderna $\left.{ }^{\circledR}\right)$. A few hours after vaccination, he developed fever, fatigue, nausea, diarrhoea and myalgia. Myalgia increased over the next few days and he presented to the emergency department 4 days after vaccination. On admission, he was afebrile $\left(37.1^{\circ} \mathrm{C}\right)$ with a blood pressure of $131 / 77$ $\mathrm{mmHg}$, a pulse of $76 \mathrm{bpm}$ and an oxygen saturation of $97 \%$ on room air. The physical examination was unremarkable with no signs of a local injection site reaction. He had mildly elevated C-reactive protein and normal white blood cell counts (Table 1). A nasopharyngeal swab was negative for SARS-CoV-2. SARS-CoV-2 serological testing was positive for nucleocapsid SARS-CoV-2 IgG/lgM (Elecsys ${ }^{\circledR}$ Anti-SARS-CoV-2 $\mathrm{N}$ Test) with a titre of 78.7 multiple of cut-off (cut-off 1.0). We suspected a severe prolonged systemic reaction to the mRNA-1273 SARSCoV-2 vaccine after natural immunization due to COVID-19 infection 6 months before, and discharged him with symptomatic therapy. The patient reported total recovery 2 days later. 


\section{DISCUSSION}

SARS-CoV-2 is an RNA virus with an envelope consisting of three protein components, and is responsible for the COVID-19 pandemic ${ }^{[1]}$. In current routine clinical practice, two types of SARS-CoV-2-specific antibodies are measured: the SARS-CoV-2 nucleocapsid and the SARS-CoV-2 spike antibodies. In case of natural immunization due to infection with SARS-CoV-2, we expect both the nucleocapsid and the spike antibodies to be positive, whereas in case of immunization with a vaccine, only spike antibodies are positive. Thus, the nucleocapsid antibodies provide information on whether natural immunization has taken place or not. For the assessment of the immune response to vaccination, measurement of the spike antibody titre before and after vaccination is essential (Fig. 2).

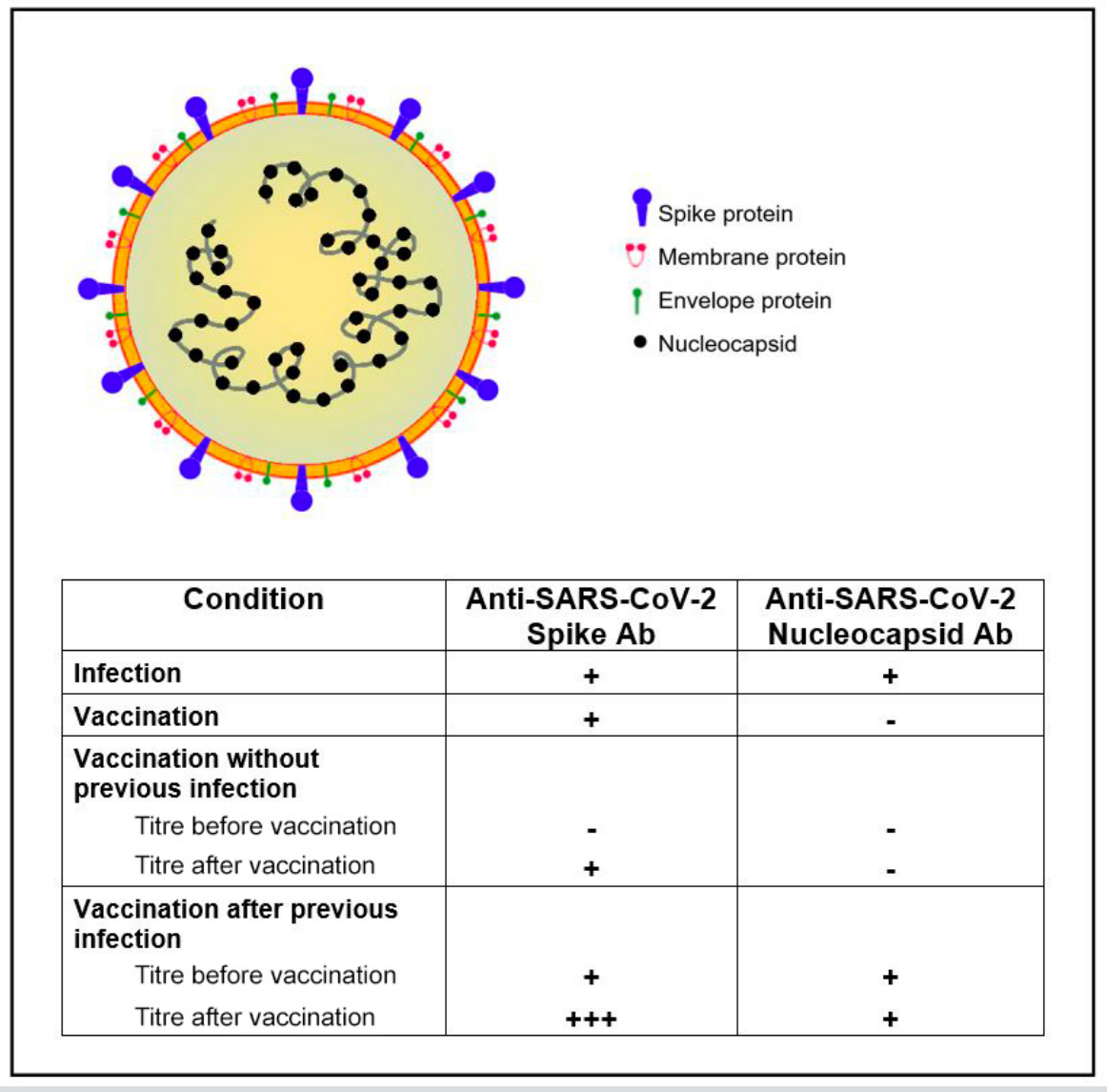

Figure 2. Simplified scheme of SARS-CoV-2 structure and interpretation of serological SARS-CoV2-antibody patterns. Ab, antibodies; +, positive test; +++, highly positive test; negative test

The patient in the reported case had a nucleocapsid SARS-CoV-2 IgG/lgM titre of 78.7 multiple of cut-off. The highly positive nucleocapsid antibody titre indicated a persistent humoral immune response 6 months after initial SARS-CoV-2 infection, corroborating findings from a recently published study ${ }^{[2]}$. Although antibody responses show a moderate decay over time, immunological memory seems to persist for up to 8 months after infection, with memory B cells actually increasing between 1 and 8 months after infection ${ }^{[2]}$.

We observed that our patient developed strong systemic symptoms after receiving the first dose of the mRNA-1273 vaccine. Symptoms consisted of fever, fatigue, nausea, diarrhoea and myalgia, which lasted for 6 days. Although immediate injection-site reactions are the most frequent adverse event of mRNA-1273 SARS-CoV-2 vaccination, systemic adverse events are common with $54.9 \%$ of patients affected and last for a mean of 2.9 days after the first vaccination dose ${ }^{[3]}$. We assume that our patient with naturally acquired immunity had a prolonged systemic reaction due to the rapid immune response triggered by the first vaccination dose. This is in line with results of a study with 110 participants showing that systemic side effects were more likely in participants with previous COVID-19 ${ }^{[4]}$. Accordingly, spike IgG titres were 10-45 times higher in patients with naturally acquired immunity in comparison with patients without pre-existing immunity after the first vaccination dose ${ }^{[4]}$. In order to quantify the immune response induced by vaccination in our patient, we should have measured the SARS-CoV-2 spike antibody titre before and after vaccination. Thus, for clinicians it is important to know that depending on the clinical situation of SARS-CoV-2 infection and/or vaccination, different antibody titres have to be determined. 


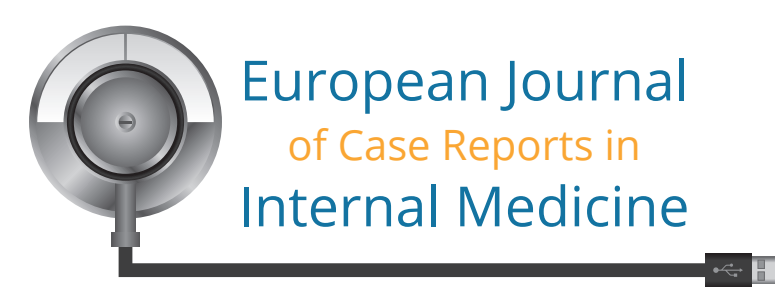

In patients with pre-existing naturally acquired immunity, no further increase in the SARS-CoV-2 spike antibody titre was noted after the second vaccine dose ${ }^{[4,5]}$, so we assume that one dose of the mRNA-1273 SARS-CoV-2 vaccine provides sufficient efficacy for our patient.

\section{REFERENCES}

1. Masters PS. The molecular biology of coronaviruses. Adv Virus Res 2006;65:193-292. doi:10.1016/S0065-3527(06)66005-3

2. Dan JM, Mateus J, KatoY,Hastie KM,YuED,FalitiCE, etal. Immunological memorytoSARS-CoV-2 assessed for up to 8 months after infection.Science 2021;371(6529):eabf4063. doi:10.1126/science.abf4063

3. Baden LR, EI Sahly HM, Essink B, Kotloff K, Frey S, Novak R, et al. Efficacy and safety of the mRNA-1273 SARS-CoV-2 vaccine. N Engl J Med 2021;384(5):403-416. doi:10.1056/ nejmoa2035389

4. Krammer F, Srivastava K, Alshammary H, Amoako AA, Awawda MH, Beach KF, et al. Antibody responses in seropositive persons after a single dose of SARS-CoV-2 mRNA vaccine. N Engl J Med 2021;384(14):1372-1374. doi:10.1056/NEJMc2101667

5. Saadat S, Rikhtegaran Tehrani Z, Logue J, Newman M, Frieman MB, Harris AD, et al. Binding and neutralization antibody titers after a single vaccine dose in health care workers previously infected with SARS-CoV-2. JAMA 2021;325(14):1467-1469. doi:10.1001/jama.2021.3341 